\title{
Extended Poroelasticity: An Analytical Solution and Its Application to p-Wave Propagation in Cervical Tissues
}

\author{
Roberto Palma ${ }^{1}{ }^{1}$ and Antonio M. Callejas ${ }^{2}$ \\ ${ }^{1}$ Department of Mechanical Engineering and Construction, Universitat Jaume I, Castellón de la Plana, Spain \\ ${ }^{2}$ Structural Mechanics and Hydraulic Engineering, University of Granada, Granada, Spain \\ Correspondence should be addressed to Roberto Palma; rpalma@uji.es
}

Received 14 September 2018; Accepted 17 October 2018; Published 29 October 2018

Guest Editor: Akhtar A. Khan

Copyright (c) 2018 Roberto Palma and Antonio M. Callejas. This is an open access article distributed under the Creative Commons Attribution License, which permits unrestricted use, distribution, and reproduction in any medium, provided the original work is properly cited.

\begin{abstract}
This work presents a semianalytical solution based on Laplace transform to study the behaviour of poroelastic materials in the context of the Extended Nonequilibrium Thermodynamics. In this framework, the fluid phase incorporates a relaxation time and, consequently, a frequency-dependence appears. This rheological behaviour could explain the frequency-dependence experimentally observed in biological tissues, which has traditionally attributed to the solid phase of tissues. In particular, the analytical solution is applied to two cases, heaviside and sinusoidal inputs, of a semi-infinite domain, which is filled with a material such as the human cervix. From the results, it is observed that the frequency-dependence of the fluid phase could be relevant to high relaxation times while for null relaxation times the classical poroelastic theory is recovered. Finally, the present analytical solution could be used to validate future computational codes and experimental settings.
\end{abstract}

\section{Introduction}

Nowadays, understanding mechanical behaviour of the human cervix is a challenge for theoretical, computational, and experimental communities since it could be used to develop functional anticipation diagnostic tools, which will be applied to reduce the main cause of infant mortality: preterm birth, according to [1]. Notice that the understanding of cervical tissues is considered one of the most pressing problems in obstetrics [2].

According to [3], the human cervix is composed of a distribution of cells embedded in an extracellular matrix of fibrillar collagen, which can be considered as the solid phase and represent $20-30 \%$ of the tissue and water with dissolved salts (fluid phase). The latter phase is the responsible for the cervix incompressibility [4].

There exist many models to study dynamic responses of tissues in specialised literature. However, most of them are contradictory since they are based on phenomenological equations, which lack robustness. In particular, measured values in cervix tissues differ several orders of magnitude for medium and high frequencies [5]. In this connection, there are several viscoelastic approaches to properly characterise the human cervix: a linear viscoelastic model was proposed in [6], an in-homogenous model in [7], and several approaches by the Rus' group; see [8-10].

Despite the fact that the cervix is composed of solid and fluid, all the previous works are only focused on the solid contribution. On the contrary, the Extended Nonequilibrium Thermodynamics (ENET) [11] incorporates a viscous-like term to the fluid phase, which could explain the frequencydependence of the cervix. For instance, R. Palma et al. have developed analytical and numerical solutions to study the second sound in thermoelasticity (see $[12,13])$, which can be considered as a viscothermal effect. Also, ENET is applied in [14] to study the Debye relaxation: a viscoelectric effect.

On this ground, this paper presents a poroelastic formulation based on the ENET to study the frequency-dependence of cervix tissues due to the fluid phase, and it could be considered the main novelty of the present work. Then, the formulation is expressed in one-dimension in order to obtain a dynamical solution by a semianalytical approach based 
on Laplace transform. Finally, two numerical examples are developed to highlight the importance of the fluid-relaxation in the response of the cervix.

The present work assumes small strains and linear elasticity for the solid phase. For the fluid, constant properties are considered and the effects of temperature are neglected.

\section{Three-Dimensional Governing Equations}

Consider an arbitrary domain $\Omega$ and boundary $\Gamma$ for which the governing equations are composed of equilibrium and constitutive equations and of the boundary conditions.

With regard to the equilibrium equation and since the domain contains solid and fluid constituents, two equations must be enforced: linear momentum and mass conservation. Mathematically, they are expressed in local form as follows:

$$
\begin{array}{r}
\rho \ddot{\mathbf{u}}=\nabla \cdot \boldsymbol{\sigma}^{\top}+\mathbf{f}, \\
c_{p u} \dot{\epsilon}+c_{p p} \dot{p}=-\nabla \cdot \mathbf{d}+D,
\end{array}
$$

where $\rho$, $\ddot{\mathbf{u}}$, and $\mathbf{f}$ denote mass density (including solid and fluid), acceleration, and body forces, respectively; $\boldsymbol{\sigma}=\boldsymbol{\sigma}^{\top}$ is the Cauchy stress tensor; $\mathbf{d}$ and $D$ denote the rate of change in fluid mass through the boundary and the production of fluid from an external source, respectively. Finally, $\epsilon, p$, $c_{p u}$, and $c_{p p}$ denote volumetric strain, fluid pressure, and two constants closely related to the porosity and fluid bulk modulus, respectively.

Obviously, two constitutive equations, solid and fluid phases, are required to model poroelasticity; these equations read

$$
\begin{aligned}
\boldsymbol{\sigma} & =\mathbf{C}: \boldsymbol{\epsilon}-c_{u p} p \mathbf{I}, \\
\tau \dot{\mathbf{d}}+\mathbf{d} & =-\mathbf{K} \cdot \nabla p .
\end{aligned}
$$

At this point, it is necessary to define all terms in (2):

(i) C denotes the elastic fourth order tensor, which is composed of matrix $\mathbf{C}_{m t}$ and of fibre $\mathbf{C}_{f b}$ (both solid phases) by the rule of mixture:

$$
\begin{array}{r}
\mathbf{C}=\xi \mathbf{C}_{m t}+(1-\xi) \mathbf{C}_{f b}, \\
\text { with : }\left\{\begin{array}{l}
\mathbf{C}_{m t}=2 \mu_{m t} \mathbf{1}+\lambda_{m t} \mathbf{I} \otimes \mathbf{I}, \\
\mathbf{C}_{f b}=2 \mu_{f b} \mathbf{1}+\lambda_{f b} \mathbf{I} \otimes \mathbf{I},
\end{array}\right.
\end{array}
$$

where $\xi=0.88$ is the percentage of matrix, 1 denotes the fourth-order identity tensor, and $\mu, \lambda$ are the Lamé parameters for matrix and fibre constituents, respectively.

(ii) $\boldsymbol{\epsilon}=(1 / 2)(\nabla \otimes \mathbf{u}+\mathbf{u} \otimes \nabla)=\nabla^{s} \mathbf{u}$ is the small strain second order tensor and $\nabla^{\mathcal{S}}$ denotes the symmetric part of the gradient of displacements.

(iii) $\mathbf{K}=\left(1 / \nu_{f}\right) \boldsymbol{\kappa}$ denotes solid permeability and it is closely related to the fluid viscosity $v_{f}$. (iv) $\tau$ is the relaxation times, which is introduced by the assumption of a mixed entropy; see [15]. This empirical parameter is responsible for viscosity in the fluid phase and, consequently, for the frequencydependence. Notice that the classical poroelasticity theory is recovered by imposing $\tau=0$.

Finally, the Dirichlet and Neumann boundary conditions for the extended poroelastic problem read

$$
\begin{array}{r}
\mathbf{u}=\overline{\mathbf{u}}, \\
\boldsymbol{\sigma}^{\top} \cdot \mathbf{n}=\mathbf{t}, \\
p=\bar{p}, \\
\mathbf{d} \cdot \mathbf{n}=d_{c},
\end{array}
$$

where $\overline{\mathbf{u}}$ and $\bar{p}$ denote prescribed displacements and pressure, respectively, and $\mathbf{t}$ and $d_{c}$ prescribed traction and fluid flux on the boundary with outward normal $\mathbf{n}$.

\section{Analytical Solution}

This section presents a one-dimensional, semianalytical, and dynamical solution for a half-space filled with a poroelastic material. For this purpose, the three-dimension extended poroelastic equations reported in Section 2 are rewritten along the $x$-axis in order to apply the state space technique; see [16]. The three-dimensional Euclidean coordinates become

$$
\mathbf{x} \equiv(x, 0,0, t) \Longrightarrow\left\{\begin{array}{l}
\mathbf{u}(\mathbf{x}, t) \equiv u(x, t) \\
p=p(x, t)
\end{array}\right.
$$

and the components of strain tensor are reduced to

$$
\{\boldsymbol{\epsilon}\} \Longrightarrow\left\{\begin{array}{l}
\epsilon_{1}=\frac{\partial u(x, t)}{\partial x}=\epsilon, \\
\epsilon_{2}=\epsilon_{3}=\epsilon_{4}=\epsilon_{5}=\epsilon_{6}=0,
\end{array}\right.
$$

and, finally, the constitutive equation of (2) (upper) becomes

$$
\begin{aligned}
& \sigma_{1}=C_{11} \epsilon_{1}-c_{u p} p \longrightarrow \sigma=C \epsilon-c_{u p} p, \\
& \sigma_{2}=\sigma_{3}=\sigma_{4}=\sigma_{5}=\sigma_{6}=0 .
\end{aligned}
$$

In absence of body forces $\mathbf{f}=\mathbf{0}$ and mass sources $D=0$, the equilibrium equations of (1) are reduced to

$$
\begin{aligned}
\frac{\partial \sigma}{\partial x} & =\rho \ddot{u}_{x} \Longrightarrow \\
\frac{\partial^{2} \sigma}{\partial x^{2}} & =\rho \frac{\partial \ddot{u}_{x}}{\partial x}=\rho \ddot{\epsilon} \\
K \frac{\partial^{2} p}{\partial x^{2}} & =\left(\frac{\partial}{\partial t}+\tau \frac{\partial^{2}}{\partial t^{2}}\right)\left(c_{p u} \epsilon+c_{p p} p\right),
\end{aligned}
$$


Now, equations (6), (7), and (8) are rewritten in the Laplace domain by applying the transformation $\bar{f}(s)=$ $\int_{0}^{\infty} f(t) e^{-s t} \mathrm{~d} t$ :

$$
\begin{aligned}
\bar{\sigma} & =C \bar{\epsilon}-c_{u p} \bar{p}, \\
\frac{\partial^{2} \bar{\sigma}}{\partial x^{2}} & =s^{2} \rho \bar{\epsilon}, \\
K \frac{\partial^{2} \bar{p}}{\partial x^{2}} & =\left(s+\tau s^{2}\right)\left(c_{p u} \bar{\epsilon}+c_{p p} \bar{p}\right) .
\end{aligned}
$$

These equations can be expressed in compact form by introducing the coefficients $L_{1}, L_{2}, M_{1}$, and $M_{2}$ :

$$
\begin{aligned}
L_{1} & =\left(\frac{c_{p u} c_{u p}+C c_{p p}}{K C}\right)\left(s+\tau s^{2}\right), \\
L_{2} & =\left(\frac{c_{p u}}{K C}\right)\left(s+\tau s^{2}\right), \\
M_{1} & =\frac{c_{u p} \rho s^{2}}{C}, \\
M_{2} & =\frac{s^{2} \rho}{C},
\end{aligned}
$$

to give

$$
\begin{aligned}
& \frac{\partial^{2} \bar{p}}{\partial x^{2}}=L_{1} \bar{p}+L_{2} \bar{\sigma}, \\
& \frac{\partial^{2} \bar{\sigma}}{\partial x^{2}}=M_{1} \bar{p}+M_{2} \bar{\sigma},
\end{aligned}
$$

and the closed solution of this system of two couple equations can be expressed as follows:

$$
\left\{\begin{array}{l}
\bar{p}(x, s) \\
\bar{\sigma}(x, s)
\end{array}\right\}=\exp \left(\sqrt{\left[\begin{array}{ll}
L_{1} & L_{2} \\
M_{1} & M_{2}
\end{array}\right]}\right)\left\{\begin{array}{l}
\bar{p}(0, s)=\bar{p}_{0} \\
\bar{\sigma}(0, s)=\bar{\sigma}_{0}
\end{array}\right\} .
$$

The solution of this system is obtained by applying the Cayley-Hamilton theorem [16] to give

$$
\left\{\begin{array}{l}
\bar{p}(x, s) \\
\bar{\sigma}(x, s)
\end{array}\right\}=\left[\begin{array}{ll}
L_{11} & L_{12} \\
L_{21} & L_{22}
\end{array}\right]\left\{\begin{array}{l}
\bar{p}_{0} \\
\bar{\sigma}_{0}
\end{array}\right\}
$$

where $\bar{p}_{0}$ and $\bar{\sigma}_{0}$ are the boundary conditions and the coefficient and are explicitly given by

$$
\begin{aligned}
& L_{11}=\frac{e^{-\sqrt{\eta_{2}} x}\left(\eta_{1}-L_{1}\right)-e^{-\sqrt{\eta_{1}} x}\left(\eta_{2}-L_{1}\right)}{\eta_{1}-\eta_{2}}, \\
& L_{22}=\frac{e^{-\sqrt{\eta_{1}} x}\left(\eta_{2}-M_{2}\right)-e^{-\sqrt{\eta_{2}} x}\left(\eta_{1}-M_{2}\right)}{\eta_{2}-\eta_{1}}, \\
& L_{12}=\frac{L_{2}\left(e^{-\sqrt{\eta_{1}} x}-e^{-\sqrt{\eta_{2}} x}\right)}{\eta_{1}-\eta_{2}}, \\
& L_{21}=\frac{M_{1}\left(e^{-\sqrt{\eta_{1}} x}-e^{-\sqrt{\eta_{2}} x}\right)}{\eta_{1}-\eta_{2}},
\end{aligned}
$$

where $\eta_{1}$ and $\eta_{2}$ are the solutions of the following characteristic equations:

$$
\begin{aligned}
\eta_{1}+\eta_{2} & =L_{1}+M_{2}, \\
\eta_{1} \eta_{2} & =L_{1} M_{2}-L_{2} M_{1} .
\end{aligned}
$$

Finally, the semianalytical solution is attained by imposing boundary conditions and by inverting the Laplace transform using Riemann-sum approximations, as in [12].

\section{Results}

This section presents two analytical solutions, called cases, in order to highlight the main features of the present formulation. For this purpose, the material properties are obtained from the literature (see Table 1), and they are real measured variables of the human cervix. In particular, Lamé parameters for fibre and matrix phases are obtained from $[8,9]$, bulk modulus of fluid from [17], solid permeability from [18], and fluid viscosity from [19]. Finally, the coefficient $c_{u p}=0.75$ obeys the composition of the cervix, namely, $80-70 \%$ of fluid phase; see [20].

4.1. Case I. For case I, the boundary conditions are assumed to be a heaviside unit step function $H(t)$ :

$$
\begin{aligned}
& \bar{p}_{0}=\frac{p_{0}}{s}, \\
& \bar{\sigma}_{0}=0 .
\end{aligned}
$$

Introducing (16) in (12) and taking into account (14), the solutions for $\bar{p}$ and $\bar{\sigma}$ in the Laplace domain read

$$
\begin{aligned}
& \bar{p}=\frac{p_{0}\left[\left(\eta_{1}-L_{1}\right) e^{-\sqrt{\eta_{2}} x}-\left(\eta_{2}-L_{1}\right) e^{-\sqrt{\eta_{1}} x}\right]}{s\left(\eta_{1}-\eta_{2}\right)}, \\
& \bar{\sigma}=\frac{p_{0} M_{1}\left(e^{-\sqrt{\eta_{1}} x}-e^{-\sqrt{\eta_{2}} x}\right)}{s\left(\eta_{1}-\eta_{2}\right)} .
\end{aligned}
$$

Furthermore, the mechanical displacement and the flux can be obtained taking into account

$$
\begin{aligned}
\bar{d} & =-K \frac{\partial \bar{p}}{\partial x}, \\
\frac{\partial^{2} \bar{\sigma}}{\partial x^{2}} & =s^{3} \rho \bar{u} \Longrightarrow \\
\bar{u} & =\frac{1}{s^{3} \rho} \frac{\partial^{2} \bar{\sigma}}{\partial x^{2}},
\end{aligned}
$$


TABLE 1: Material properties of the human cervix.

\begin{tabular}{lcc}
\hline Magnitude & Value & Units \\
\hline$\rho$ & 1000 & {$\left[\mathrm{~kg} / \mathrm{m}^{3}\right]$} \\
$\rho_{f}$ & 1000 & {$\left[\mathrm{~kg} / \mathrm{m}^{3}\right]$} \\
$c_{u p}$ & 0.75 & {$[-]$} \\
$v_{f}$ & $1.2 \times 10^{-3}$ & {$[\mathrm{~Pa} \cdot \mathrm{s}]$} \\
$\mu_{f b}$ & $6.45 \times 10^{7}$ & {$[\mathrm{~Pa}]$} \\
$\lambda_{f b}$ & $1.47 \times 10^{8}$ & {$[\mathrm{~Pa}]$} \\
$\mu_{m t}$ & $7.47 \times 10^{3}$ & {$[\mathrm{~Pa}]$} \\
$\lambda_{m t}$ & $1.7 \times 10^{9}$ & {$[\mathrm{~Pa}]$} \\
$B_{f}$ & $2.2 \times 10^{9}$ & {$[\mathrm{~Pa}]$} \\
$\kappa$ & $7.2 \times 10^{-14}$ & {$\left[\mathrm{~m}^{2}\right]$} \\
\hline
\end{tabular}

to give

$$
\begin{aligned}
& \bar{d} \\
& =\frac{K p_{0}\left[\left(\eta_{1}-L_{1}\right) \sqrt{\eta_{2}} e^{-\sqrt{\eta_{2}} x}-\left(\eta_{2}-L_{1}\right) \sqrt{\eta_{1}} e^{-\sqrt{\eta_{1}} x}\right]}{s\left(\eta_{1}-\eta_{2}\right)}, \\
& \bar{u}=\frac{p_{0} M_{1}\left(\eta_{1} e^{-\sqrt{\eta_{1} x}}-\eta_{2} e^{-\sqrt{\eta_{2}} x}\right)}{\rho s^{4}\left(\eta_{1}-\eta_{2}\right)} .
\end{aligned}
$$

Consider a one-dimensional and semi-infinite domain, which is filled with a material as that of cervix. At $t=$ 0 , a fluid pressure of heaviside type is applied and, then, the evolution of all variables of (17) and (18) is numerically studied. In particular, Figure 1 shows the time evolution of $p$ (left column), $u$ (middle column), and $\sigma$ (right column) for three different relaxation times: $\tau=0.5,0,1,0[\mathrm{~s}]$ (each one in a row). In addition, each figure contains three different curves at three time instants $t=0.3,0.5,1[\mathrm{~s}]$.

For $\tau \neq 0[s]$ (extended poroelasticity) a hyperbolic behaviour is attained in the evolution of $p$ and, consequently, in $u$. It is observed in the wave front, which is moving at each time instant. On the contrary, for the case $\tau=0 \mathrm{a}$ parabolic behaviour is observed, absence of wave front, and the classical poroelastic theory is recovered. In this sense, the extended poroelasticity removes the paradox of infinite velocity, which lacks physical meaning: in nature, there are no infinity velocities.

Since the problem is coupled, the stresses for $\tau \neq 0$ [s] show a viscous-like behaviour that could explain the frequency-dependence experimentally observed in cervical tissues. On the contrary, for $\tau=0[\mathrm{~s}]$, a linear behaviour is observed and, therefore, it is concluded that the classical poroelasticity can not be used to model the human cervix.

4.2. Case II. For case II, a sinusoidal function $p_{0} \sin (\omega t)$ of frequency $\omega$ is prescribed:

$$
\begin{aligned}
& \bar{p}_{0}=p_{0} \frac{\omega}{s^{2}+\omega^{2}}, \\
& \bar{\sigma}_{0}=0 .
\end{aligned}
$$

Following a procedure similar to that of case I, the solutions for $\bar{p}$ and $\bar{\sigma}$ in the Laplace domain are

$$
\begin{aligned}
& \bar{p}=\frac{p_{0} \omega\left[\left(\eta_{1}-L_{1}\right) e^{-\sqrt{\eta_{2}} x}-\left(\eta_{2}-L_{1}\right) e^{-\sqrt{\eta_{1}} x}\right]}{\left(s^{2}+\omega^{2}\right)\left(\eta_{1}-\eta_{2}\right)}, \\
& \bar{\sigma}=\frac{p_{0} M_{1} \omega\left(e^{-\sqrt{\eta_{1}} x}-e^{-\sqrt{\eta_{2}} x}\right)}{\left(s^{2}+\omega^{2}\right)\left(\eta_{1}-\eta_{2}\right)},
\end{aligned}
$$

and the mechanical displacement and the flux are given by

$\bar{d}$

$$
\begin{aligned}
& =\frac{K p_{0} \omega\left[\left(\eta_{1}-L_{1}\right) \sqrt{\eta_{2}} e^{-\sqrt{\eta_{2}} x}-\left(\eta_{2}-L_{1}\right) \sqrt{\eta_{1}} e^{-\sqrt{\eta_{1}} x}\right]}{\left(s^{2}+\omega^{2}\right)\left(\eta_{1}-\eta_{2}\right)}, \\
& \bar{u}=\frac{p_{0} M_{1} \omega\left(\eta_{1} e^{-\sqrt{\eta_{1}} x}-\eta_{2} e^{-\sqrt{\eta_{2}} x}\right)}{\rho s^{3}\left(s^{2}+\omega^{2}\right)\left(\eta_{1}-\eta_{2}\right)} .
\end{aligned}
$$

Consider the same semi-infinite domain as that in case I. Now, a fluid pressure of sinusoidal type is applied at $t=0$ and all variables of (21) and (22) are shown in Figure 2. Again, the same conclusion as those in case I can be observed for this sinusoidal input. Nevertheless, the curves are smoother due to the nature of the sinusoidal signal. Therefore, these solutions are more amenable for future computational validations since it is not necessary to use regularisation schemes.

\section{Conclusions}

This work has presented a theoretical approach based on Nonequilibrium Thermodynamics to study the behaviour of poroelastic materials taking into account the frequencydependence of the fluid phase. In this connection, the main novelty of the present work is the incorporation of relaxation times for the fluid phase to perform a material constitution applied to biological tissues. Then, the three-dimensional governing equations are reduced to one dimension in order to obtain a semianalytical and dynamical solution based on Laplace transform. In particular, the solution is applied to simulate a semi-infinite domain, which is filled with a material such as the human cervix, and it is observed that the frequency-dependence also could be due to the fluid phase. 

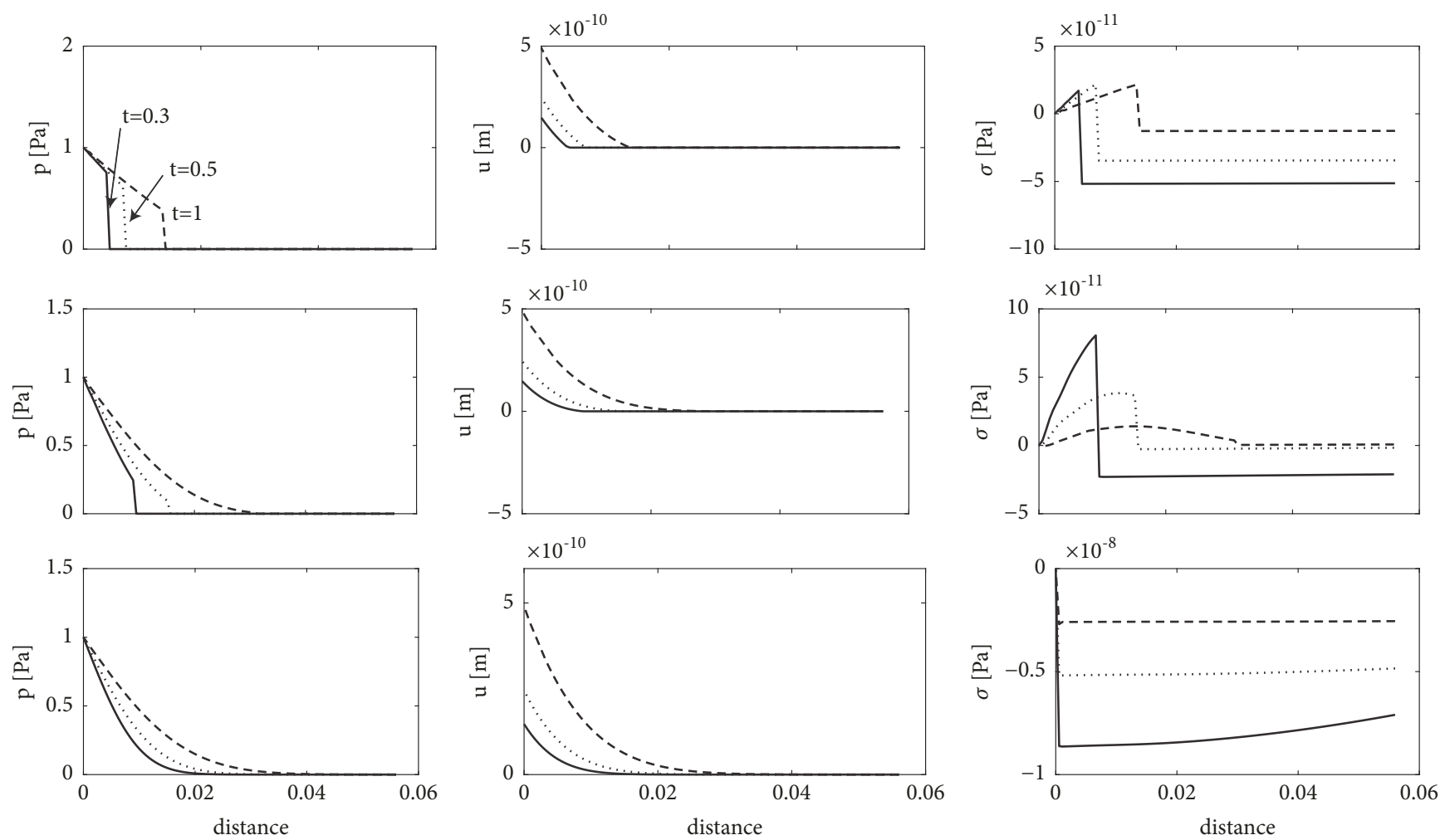

FIGURE 1: Case I. Fluid pressure (left column), mechanical displacement (middle), and mechanical stress (right) versus distance for three relaxation times: $\tau=0.5$ (top row), $\tau=0.1$ (middle) and $\tau=0[\mathrm{~s}]$ (bottom). Each figure shows three different curves at three time instants: $t=0.3,0.5,1[\mathrm{~s}]$.
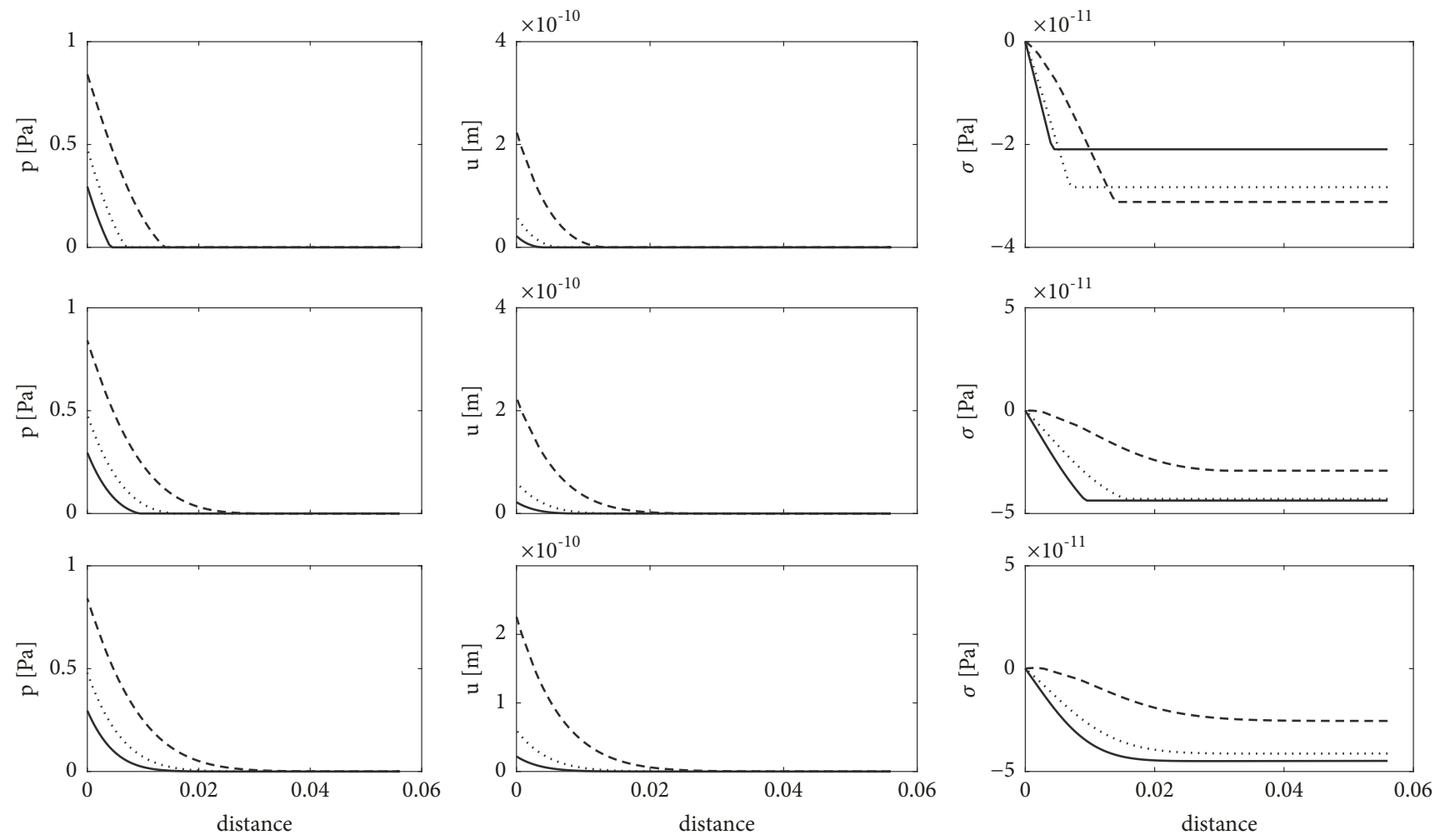

Figure 2: Case II. Fluid pressure (left column), mechanical displacement (middle), and mechanical stress (right) versus distance for three relaxation times: $\tau=0.5$ (top row), $\tau=0.1$ (middle), and $\tau=0[\mathrm{~s}]$ (bottom). Each figure shows three different curves at three time instants: $t=0.3,0.5,1[\mathrm{~s}]$. 


\section{Data Availability}

The data used to support the findings of this study are available from the corresponding author upon request.

\section{Conflicts of Interest}

The authors declare that they have no conflicts of interest.

\section{Acknowledgments}

This research was supported by the Ministry of Education DPI2017-83859- R, DPI2014-51870- R, DPI2010-17065, and UNGR15-CE- 3664, Ministry of Health DTS15/00093, and PI16/00339, PI-0107-2017, PIN-0030-2017, and Junta de Andalucía P11-CTS- 8089 projects.

\section{References}

[1] M. House and S. Socrate, "The cervix as a biomechanical structure," Ultrasound in Obstetrics \& Gynecology, vol. 28, no. 6, pp. 745-749, 2006.

[2] N. Uldbjerg, G. Ekman, A. Malmström, B. Sporrong, U. Ulmsten, and L. Wingerup, "Biochemical and morphological changes of human cervix after local application of prostaglandin e2 in pregnancy," The Lancet, vol. 31, pp. 267-268, 1981.

[3] M. Winkler and W. Rath, "Changes in the cervical extracellular matrix during pregnancy and parturition," Journal of Perinatal Medicine, vol. 27, no. 1, pp. 45-60, 1999.

[4] S. Weiss, T. Jaermann, P. Schmid et al., "Three-dimensional fiber architecture of the nonpregnant human uterus determined ex vivo using magnetic resonance diffusion tensor imaging," Anatomical Record - Part A Discoveries in Molecular, Cellular, and Evolutionary Biology, vol. 288, no. 1, pp. 84-90, 2006.

[5] E. Turgay, S. Salcudean, and R. Rohling, "Identifying the mechanical properties of tissue by ultrasound strain imaging," Ultrasound in Medicine \& Biology, vol. 32, no. 2, pp. 221-235, 2006.

[6] J. M. Pereira, J. M. Mansour, and B. R. Davis, "Dynamic measurement of the viscoelastic properties of skin," Journal of Biomechanics, vol. 24, no. 2, pp. 157-162, 1991.

[7] E. A. Barannik, A. Girnyk, V. Tovstiak, A. I. Marusenko, S. Y. Emelianov, and A. P. Sarvazyan, "Doppler ultrasound detection of shear waves remotely induced in tissue phantoms and tissue in vitro," Ultrasonics, vol. 40, no. 1-8, pp. 849-852, 2002.

[8] L. Peralta, G. Rus, N. Bochud, and F. S. Molina, "Assessing viscoelasticity of shear wave propagation in cervical tissue by multiscale computational simulation," Journal of Biomechanics, vol. 48, no. 9, pp. 1549-1556, 2015.

[9] L. Peralta, G. Rus, N. Bochud, and F. S. Molina, "Mechanical assessment of cervical remodelling in pregnancy: Insight from a synthetic model," Journal of Biomechanics, vol. 48, no. 9, pp. 1557-1565, 2015.

[10] A. Callejas, A. Gomez, J. Melchor et al., "Performance Study of a Torsional Wave Sensor and Cervical Tissue Characterization," Sensors, vol. 17, no. 9, p. 2078, 2017.

[11] D. Jou, J. Casas-Vázquez, and G. Lebon, Extended Irreversible Thermodynamics, Springer-Verlag, Berlin, Heidelberg, Germany, 1996.

[12] R. Palma, J. L. Pérez-Aparicio, and R. L. Taylor, "Non-linear finite element formulation applied to thermoelectric materials under hyperbolic heat conduction model," Computer Methods Applied Mechanics and Engineering, vol. 213-216, pp. 93-103, 2012.

[13] R. Palma, E. Moliner, and J. L. Pérez-Aparicio, "Elastothermoelectric beam formulation for modeling thermoelectric devices," Finite Elements in Analysis and Design, vol. 129, pp. 3241, 2017.

[14] R. Palma, J. L. Perez-Aparicio, and R. L. Taylor, "Dissipative finite element formulation applied to piezoelectric materials with Debye memory," IEEE/ASME Transactions on Mechatronics, 2018.

[15] G. Lebon, T. Desaive, and P. Dauby, "A unified extended thermodynamic description of diffusion, thermo-diffusion, suspensions, and porous media," Transaction of the ASME, vol. 73, no. 1, pp. 16-20, 2006.

[16] H. M. Youssef and E. A. Al-Lehaibi, "State-space approach of two-temperature generalized thermoelasticity of onedimensional problem," International Journal of Solids and Structures, vol. 44, no. 5, pp. 1550-1562, 2007.

[17] J. B. Pendry and J. Li, "An acoustic metafluid: Realizing a broadband acoustic cloak," New Journal of Physics, vol. 10, 2008.

[18] F. J. O’Brien, B. A. Harley, M. A. Waller, I. V. Yannas, L. J. Gibson, and P. J. Prendergast, "The effect of pore size on permeability and cell attachment in collagen scaffolds for tissue engineering," Technology and Health Care, vol. 15, no. 1, pp. 3-17, 2007.

[19] L. Colla, L. Fedele, M. Scattolini, and S. Bobbo, "Water-based Fe $2 \mathrm{O} 3$ nanofluid characterization: Thermal conductivity and viscosity measurements and correlation," Advances in Mechanical Engineering, vol. 2012, 2012.

[20] P. Fratzl, “Collagen: structure and mechanics, an introduction," Collagen: Structure and Mechanics, pp. 1-13, 2008. 


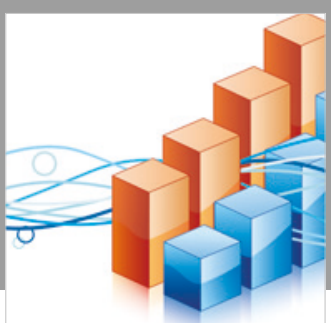

Advances in

Operations Research

\section{-n-m}
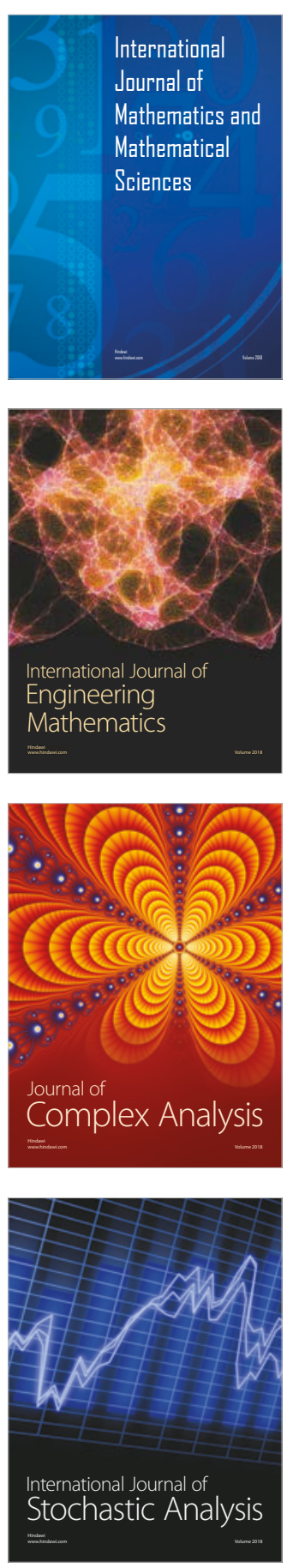
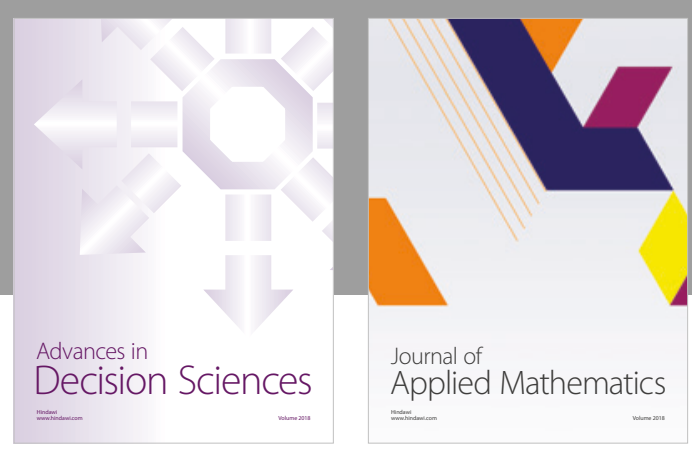

Journal of

Applied Mathematics
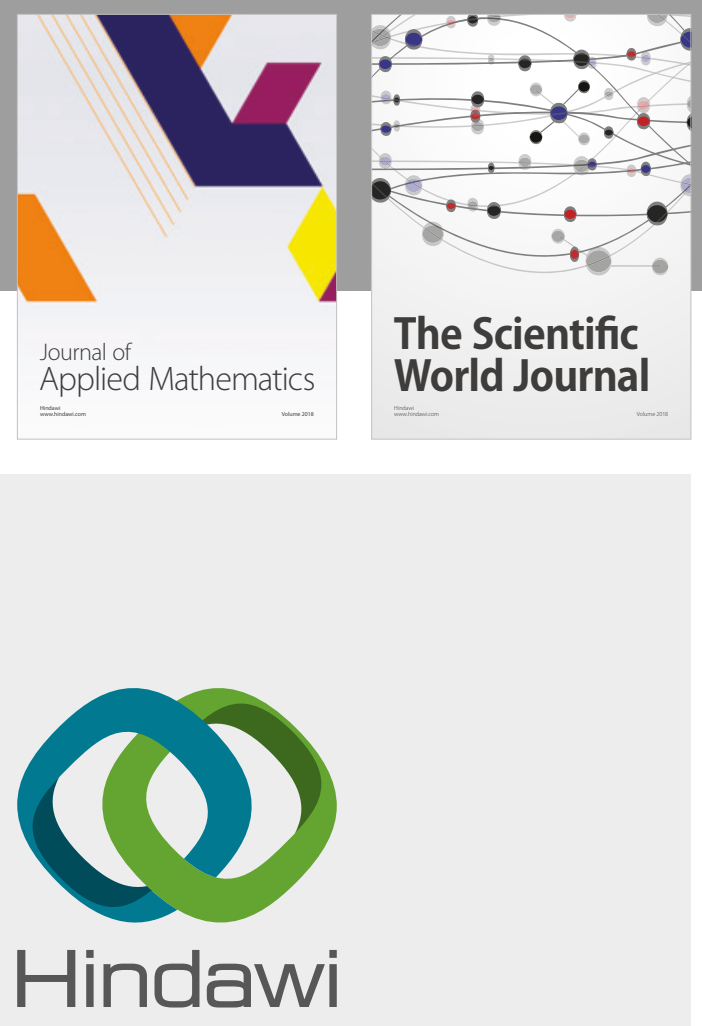

Submit your manuscripts at

www.hindawi.com

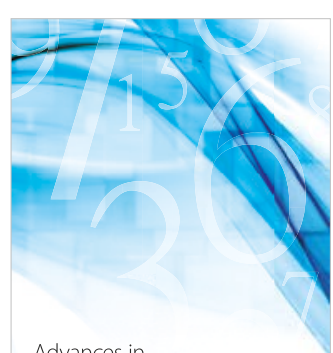

Advances in
Numerical Analysis
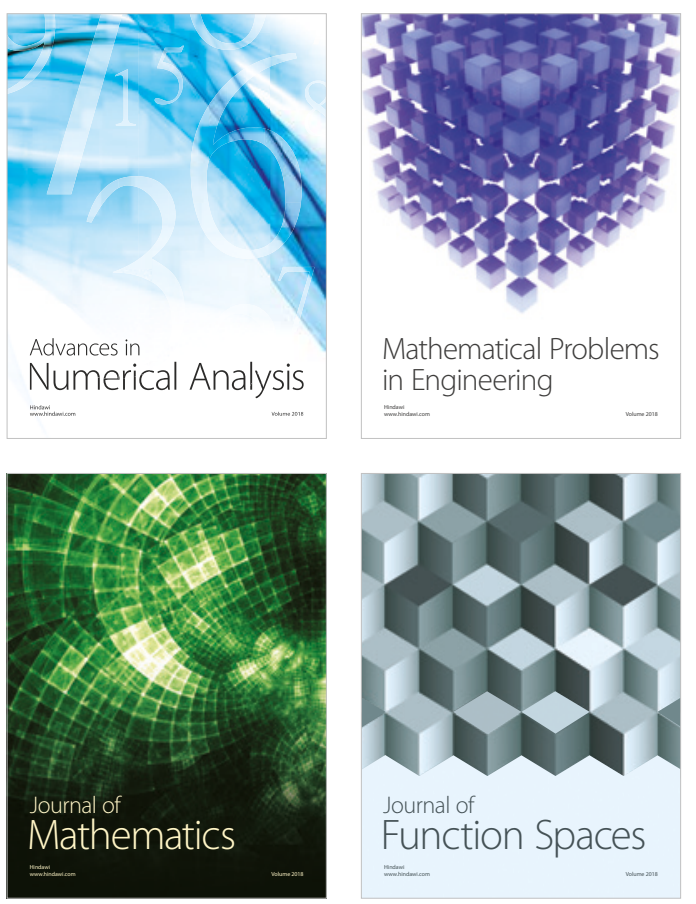

Mathematical Problems in Engineering

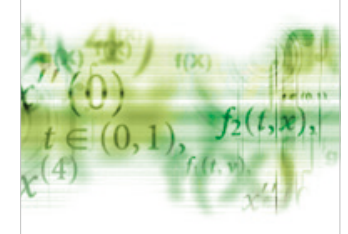

International Journal of

Differential Equations

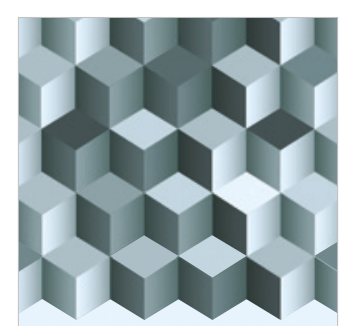

Journal of

Function Spaces

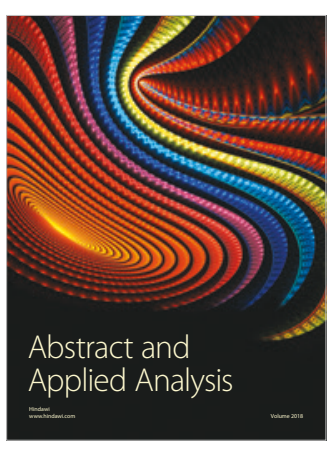

The Scientific

World Journal

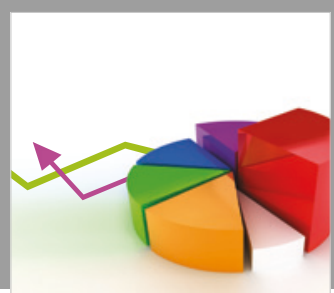

Journal of

Probability and Statistics
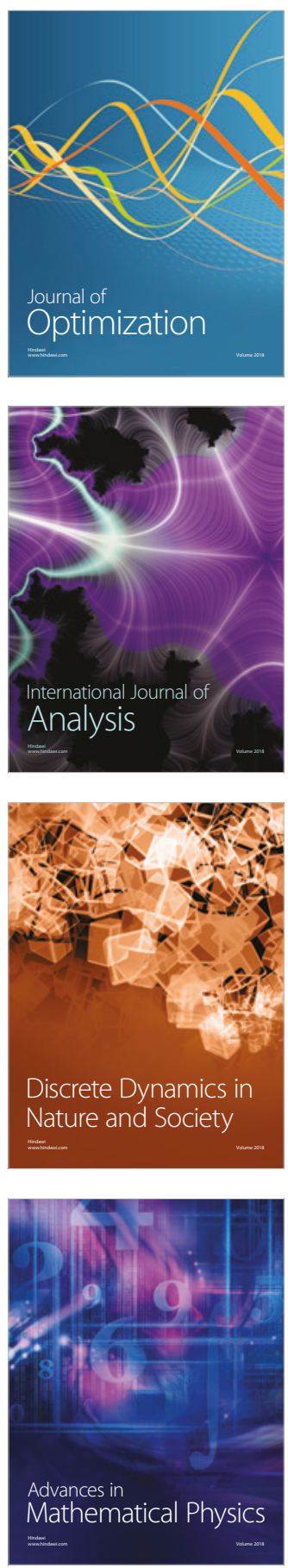